\title{
MRNA Export
}

National Cancer Institute

\section{Source}

National Cancer Institute. mRNA Export. NCI Thesaurus. Code C19424.

Transport of messenger RNA from its nuclear site of transcription to the cytoplasm, where the message is translated into protein by ribosomes. 